\title{
Comparación de la cinética de remoción de la lignina de bagazos de caña de azúcar (Saccharum Officinarum) y cactácea (Opuntia Ficus-Indica), utilizando enzima lacasa
}

\author{
Kinetic Comparison of Lignin Removal from Sugarcane (Saccharum Officinarum) \\ and Cactus (Opuntia Ficus-Indica) Bagasse by using Laccase Enzyme
}

\author{
Nubia Rosa Cardona López ${ }^{1}$, James Alberto Marín², Oscar Julio Medina Vargas ${ }^{3}$, Luis Alberto Ríos ${ }^{4}$ y \\ Gerardo Andrés Caicedo Pineda ${ }^{5}$
}

\begin{abstract}
Resumen
El objetivo de este estudio fue evaluar el efecto de la enzima lacasa proveniente de Aspergillus sp., para exponer la celulosa en los bagazos de caña de azúcar (Saccharum officinarum) (BCA) y cactácea (Opuntia-ficus) (BOF). Para ello, se llevó a cabo la determinación de los parámetros cinéticos de la enzima en cada uno de los materiales, en términos de velocidades iniciales, a diversas proporciones bagazo:solución (10 g:L a $50 \mathrm{~g}: \mathrm{L})$, con un tiempo total de reacción de $1 \mathrm{~h}$. Bajo las condiciones de reacción evaluadas, se evidenció remoción de extraíbles; sin embargo, se considera que esto no fue debido a la acción enzimática, sino por efecto del búffer. Por otra parte, no hubo disolución de celulosa ni hemicelulosa, pero sí de lignina. Por lo tanto, los resultados se analizaron teniendo en cuenta la ecuación de Michaelis-Menten, utilizando como base la tasa de desaparición de la proporción $g_{\text {lignina }}:$ L. Por medio de una linealización de Lineweaver-Burk, se obtuvo el mejor ajuste de valores, donde $V_{\text {máx }}=$ $14.9566 g_{\text {lignina }}: \mathrm{L} / \mathrm{h}$ y $k_{m}=40.2011 g_{\text {lignina }}: \mathrm{L}$ para el BCA $(\mathrm{R}=0.9933)$ y $V_{\text {máx }}=26.8300 g_{\text {lignina }}: \mathrm{L} / \mathrm{h}$ y $k_{m}=43.5575 g_{\text {lignina }}: \mathrm{L}$ para el BOF $(\mathrm{R}=0.9972)$. Con base en lo anterior, aunque la lacasa presentó una afinidad enzimática similar para ambos bagazos, se predice una mejor eficiencia catalítica hacia la remoción de lignina de BOF (0.6160).
\end{abstract}

Palabras clave: bagazo de caña, bagazo de cactáceas, lignina, celulosa, lacasa, cinética enzimática

\begin{abstract}
The present study evaluates the effect of a laccase from Aspergillus sp. to expose cellulose contained in both sugarcane (Saccharum officinarum, BCA) and Opuntia-ficus cactus (BOF) bagasse. The kinetic parameters of the enzymatic activity were calculated for each of the materials, using the initial rates method at various bagasse:solution ratios (10 g:L to $50 \mathrm{~g}: \mathrm{L})$ and a total reaction time of $1 \mathrm{~h}$. The assays presented a non-enzymatic removal of extractables by the buffer effect, under the evaluated reaction conditions. On the other hand, there was no cellulose or hemicellulose dissolution but there was lignin. Therefore, the results were analyzed, considering the Michaelis-Menten equation and using the disappearance rate of the $g_{\text {lignin }}: \mathrm{L}$ ratio as a basis. Through a LineweaverBurk linearization, the best fit of the values was obtained, where $V_{\max }=14.9566 g_{\text {lignin }}: \mathrm{L} / \mathrm{h}$ and $k_{m}=40.2011 g_{\text {lignin }}: \mathrm{L}$ for BCA assays $(\mathrm{R}=0.9933)$ and $V_{\max }=26.8300 g_{\text {lignin }}: \mathrm{L} / \mathrm{h}$ and $k_{m}=43.5575 g_{\text {lignin }}: \mathrm{L}$ for BOF assays $(\mathrm{R}=0.9972)$. Based on the above, although laccase presented a similar enzymatic affinity for both bagasse, a better catalytic efficiency is predicted towards lignin removal from BOF (0.6160).
\end{abstract}

Keywords: sugarcane bagasse, cactus bagasse, lignin, cellulose, laccase, enzymatic kinetics

Recepción: 10-nov-2020

Aceptación: 11-dic-2020

\footnotetext{
${ }^{1}$ Universidad Pedagógica y Tecnológica de Colombia, Procesos Ambientalmente Amigables, Tunja, Colombia. Corresponding author: nubia. cardona@uptc. edu. co

${ }^{2}$ Universidad de Antioquia, Grupo Procesos Químicos Industriales, Medellín, Antioquia.

${ }^{3}$ Universidad Pedagógica y Tecnológica de Colombia, Grupo de Investigación en Química y Tecnología de Alimentos, Tunja, Colombia.

${ }^{4}$ Universidad de Antioquia, Grupo Procesos Químicos Industriales, Medellín, Antioquia.

${ }^{5}$ Universidad Pedagógica y Tecnológica de Colombia, Procesos Ambientalmente Amigables, Tunja, Colombia.
} 


\section{Introducción}

En la actualidad, la biomasa lignocelulósica se ha convertido en un recurso potencial renovable, que llama la atención para la purificación del material celulósico, donde los subproductos agroindustriales con mayor contenido de este polímero corresponden al bagazo de caña, el cual está abundantemente disponible y tiene una alta concentración de celulosa (aproximadamente 40\% - 50\%) [1]. De acuerdo con el Atlas de potencial energético de BoyacáColombia, se producen cerca de 161627 t/año de caña de azúcar, de los cuales 1015018 t/año son residuos en forma de bagazo, producido durante la etapa de recolección y procesamiento en esta zona. La extensiva explotación agrícola causa generación de residuos que carecen de importancia económica, contaminación en los suelos por lixiviados, crecimiento de microorganismos que pueden afectar negativamente el producto final $\mathrm{y}$ emisión de gases por el efecto invernadero. A su vez, una disminución de la calidad del suelo afecta sus propiedades físicas y químicas, causándole una degradación y erosión que inhibe la producción agrícola [2, 3].

Entre otros productos de Boyacá se encuentran las cactáceas Opuntia ficus-indica, conocida por su producción de hidrocoloide o mucílago, a partir de los cladodios y los frutos. Este mucílago se puede utilizar como aditivo en la industria de alimentos, por su propiedad espesante, su gran capacidad de adsorción de agua, de formar geles, como reemplazante de grasas en diversos alimentos, entre otras aplicaciones [4-6]. El mayor contenido de mucilago se encuentra en el material vegetal de la planta y los frutos, por lo que la cáscara en forma de bagazo es descartada sin uso alguno. Sin embargo, aunque no se conoce en su totalidad la composición química del residuo, tiene potencial de utilizarse como material celulósico, debido a que es uno de los componentes principales del mismo [7].

La celulosa se considera una fuente natural casi inagotable y con potencial de aplicaciones industriales, principalmente para la elaboración de papel y muebles, una variedad se utiliza en las industrias farmacéutica (bioadhesivos para formulaciones bucales, oculares y nasales), biomédica (membranas de purificación de sangre), energética (producción de biocombustibles) y alimentaria (postres tradicionales y envasados hidrofóbicos y antimicrobianos de alimentos [8-16]. El material celulósico proveniente de estos residuos presentan un ordenamiento estructural, en el que sus grupos hidroxilos generan fuertes uniones intramoleculares, adquiriendo propiedades cristalinas y propiedades adecuadas para productos ecológicos, que tienen una gran demanda [17].

Las materias primas fibrosas deben reunir determinados requisitos de índole técnica y económica, donde los más importantes radican en la composición química del material, su reactividad frente a los agentes de pulpeo y sus propiedades morfológicas. Los bagazos, como residuos de industrias como la azucarera, además de satisfacer dichos requerimientos, se encuentran disponible en grandes cantidades, en donde solo una mínima fracción es utilizada de manera directa por el hombre y/o animales, en su mayoría esta materia orgánica se transforma en residuos no comestibles que se constituyen en una fuente de contaminación ambiental. La manipulación, transportación y su almacenamiento disminuyen los riesgos de inversión por el bajo costo que requieren para dichas actividades y lo hacen un material atractivo, en comparación con otras fuentes de materia prima lignocelulósicas. Por ejemplo, una tonelada de azúcar refinada produce dos toneladas de bagazo $[18,19]$.

Actualmente, en la separación de celulosa de los componentes lignocelulósicos, se destacan los métodos químicos, los cuales garantizan su obtención en forma pura. Dichos procesos implican utilizar pretratamientos con ácidos fuertes, como el ácido sulfúrico, ácido clorhídrico, ácido fosfórico, entre otros, y tratamientos alcalinos, que utilizan hidróxido de sodio e hidróxido de potasio [20]. El pretratamiento representa la etapa de mayor costo en el fraccionamiento de la biomasa, debido a que requieren de condiciones altas de temperatura y alta concentración de los reactivos, generando residuos tóxicos y corrosivos, además, en el pretratamiento también se pierden algunos azucares fermentables de interés en la producción de bioetanol. Esto debido a la formación de componentes inhibitorios. Por lo tanto, las investigaciones recientes pretenden desarrollar 
procesos de purificación, a partir de tecnologías sostenibles y metodologías de innovación, que mejoren los procesos convencionales. Entre ellos la utilización de enzimas con la capacidad de separación de la celulosa puede ser una alternativa frente a los tratamientos químicos, ya que no se requiere altas condiciones de temperatura y presión, no generan residuos contaminantes y los resultados de extracción son comparables con los que brindan los tratamientos químicos [21].

Los usos de las enzimas van desde la industria textil hasta la industria de la pulpa y el papel y desde aplicaciones alimentarias hasta procesos de biorremediación. La enzima lacasa tiene un potencial de aplicación en bio-blanqueadores, ya que actúa directamente sobre la lignina y pueden oxidar el polímero fenólico para producir agua, utilizando oxígeno molecular como aceptor final de electrones, que es más probable que logre una producción industrial a gran escala. El empleo de enzima lacasa se debe principalmente a que polimerizan la lignina por acoplamiento de los radicales fenoxi producidos por la oxidación de los grupos fenólicos de la lignina [22]. Además, presentan una serie de ventajas frente a los pretratamientos fisicoquímicos como son la mínima producción de residuos, la generación de coproductos de valor agregado, la no generación de compuestos tóxicos que puedan comprometer la fermentación posterior y el bajo requerimiento energético [23, 24].

Por lo tanto, es importante determinar cuál sería el potencial de la enzima frente a unos sustratos específicos, con el fin de determinar si es factible o no su uso. Para ello, se puede partir de un análisis preliminar de la velocidad de reacción teniendo en cuenta los modelos que se proponen en literatura y qué variables son incidentes en el proceso. Algunos reportes indican que la acción enzimática normalmente tiene una tendencia de Michaelis-Menten, donde la concentración de los componentes lignocelulósicos juega un papel crucial al finalizar cada tratamiento, principalmente para identificar dónde actúa la enzima. Aunque la enzima lacasa puede oxidar subunidades fenólicas, es incapaz de oxidar subunidades no fenólicas con un alto potencial redox en la lignina [25].
El bagazo de caña de azúcar y de cactáceas son residuos en gran parte desechables, presentes en el departamento de Boyacá (Colombia), los cuales son menos explotados que otros residuos agrícolas. Sin embargo, pueden utilizarse como sustratos potenciales para la obtención de celulosa para aplicaciones industriales [26]. Teniendo en cuenta lo anterior, el presente trabajo muestra la evaluación de un tratamiento de dichas materias primas, utilizando la enzima lacasa, a la hora de exponer el biopolímero de la celulosa. Para ello, se analizaron los datos composicionales de las muestras, con el fin de observar qué factores inciden o no sobre el comportamiento cinético.

\section{Materiales y métodos}

\subsection{Materia prima}

Las muestras de BCA y BOF fueron suministradas por agricultores de Boyacá-Colombia. Cada uno de los sustratos fue lavado y desinfectado con una solución de hipoclorito de sodio $20 \mathrm{ppm}$ y secado a temperatura ambiente. Posteriormente, los materiales se molieron a un tamaño de partícula por debajo de $0.5 \mathrm{~mm}$ (-30 \#Ty) y se almacenaron en bolsas herméticas, para evitar el efecto de la humedad libre.

La composición química de los residuos de $\mathrm{BCA}$ y BOF se determinó siguiendo los métodos estándar de la Asociación Técnica de la Industria de Pulpa y Papel (TAPPI del inglés), de la siguiente manera: TAPPI T222 om-98 (lignina) [27], TAPPI T202 om-88 (celulosa) [28], TAPPI 211 om-85 (cenizas) y TAPPI 204-cm-97 (extraíbles) y hemicelulosa, utilizando el método de clorito de sodio [29].

\subsection{Estudio cinético y determinación de los parámetros cinéticos en el BCA y BOF}

Se prepararon ensayos a nivel de erlenmeyer, suspendiendo 1 a 5 gramos de BCA o BOF en $100 \mathrm{~mL}$ de buffer fosfato de amonio, con una concentración de $50 \mathrm{mM}$, la cual contenía $1.5 \mathrm{~mL} / \mathrm{L}$ de lacasa de Aspergillus sp. SAE0050 de SigmaAldrich, con una actividad de $1000 \mathrm{LAMU} / \mathrm{mL}$. Las condiciones del proceso fueron $\mathrm{pH} 6.0$, temperatura de $35^{\circ} \mathrm{C}$, tiempo de reacción de 1 h y $150 \mathrm{rpm}$ de 
agitación, utilizando un agitador orbital. Todos los experimentos se prepararon por triplicado, más un control negativo, sin agregar enzima.

Al finalizar los ensayos, se evaluó -el porcentaje de remoción de hemicelulosa, celulosa y lignina de las muestras tratadas, siguiendo los métodos estándar TAPPI que se utilizaron para el análisis de las muestras sin tratamiento. Los resultados fueron analizados por el método de las velocidades iniciales, teniendo en cuenta el modelo de Michaelis-Menten, utilizando la proporción lignina:solución (g:L). Para ello, se utilizaron los métodos de linealización de Lineweaver-Burk (ecuación 1), Eadie-Hofstee (ecuación 2) y Agustinson (ecuación 3), de donde se escogieron los valores de $V_{\max }$ y $k_{m}$ que mejor fueron ajustados.

$$
\begin{gathered}
\frac{1}{V_{0}}=\frac{K_{m}}{V_{\max } *[S]}+\frac{1}{V_{\max }} \\
V_{0}=V_{\max }-K_{m} \frac{V_{0}}{[S]} \\
\frac{[S]}{V_{0}}=\frac{K_{m}}{V_{\max }}+\frac{1}{V_{\max }}[S]
\end{gathered}
$$

\section{Resultados y discusión}

\subsection{Caracterización química del material celulósico de residuos BCA y BOF}

La Tabla 1 reporta los resultados obtenidos de la composición química de los residuos BCA y BOF. El porcentaje de celulosa del BCA muestra similitudes con los reportados por Mattoso et al., [30], debido a que reporta valores de $43 \%$ y $27 \%$ de celulosa y lignina, respectivamente. Así mismo, la composición química de BOF muestra analogías con los valores encontrados por otros autores, quienes indican que los cactus contienen entre un $26 \%$ y $32 \%$ de celulosa $[31,32]$. Por lo tanto, las muestras evaluadas representan un potencial de aplicación para elaboración de nuevos materiales.

Por otra parte, se observa que la composición química de cada una de las muestras depende de la fuente de procedencia del cultivo. Las diferencias entre la composición de lignina y hemicelulosa son alrededor del $2 \%$ entre ambos bagazos, siendo
Tabla 1. Caracterización de BCA y BOF

\begin{tabular}{lcc}
\hline Porcentajes & BCA & BOF \\
\hline Extraíbles orgánicos & $9.2519 \pm 0.3421$ & $26.2201 \pm 0.4309$ \\
Lignina & $30.5312 \pm 0.2732$ & $28.8421 \pm 0.3343$ \\
Hemicelulosa & $15.0531 \pm 0.4245$ & $12.7841 \pm 0.2265$ \\
Celulosa & $45.1638 \pm 0.1835$ & $32.1532 \pm 0.4523$ \\
\hline
\end{tabular}

en BCA ligeramente mayores. Sin embargo, el contenido de extraíbles (otros compuestos orgánicos) en BOF es casi 3 veces mayor. Esto puede deberse a que este material contiene cenizas, pectinas y mucilagos remanentes [7, 33].

\subsection{Estudio cinético y determinación de los parámetros cinéticos en el BCA y BOF}

La Figura 1 presenta el porcentaje de remoción de lignina, hemicelulosa, celulosa y extraíbles en los ensayos con BCA y BOF, luego del tratamiento enzimático. Se pudo observar que tanto la celulosa como la hemicelulosa, no sufrieron disolución por la acción enzimática en ninguno de los casos. Caso contrario ocurre con la lignina, la cual presentó una remoción inversamente proporcional a la relación bagazo:solución en los ensayos con ambas muestras, donde con BCA estuvo en el rango de $26 \%$ y $28 \%$ y en el caso de BOF entre $25 \%$ y $35 \%$.

Por otra parte, pudo apreciarse remoción considerable de los extraíbles en los ensayos con BCA, alrededor del $95 \%$ en todos los casos, mientras que en los ensayos con BOF alcanzó un $60 \%$. Teniendo en cuenta que en los controles negativos no se observó evidencia de remoción de celulosa, hemicelulosa y lignina, pero sí de extraíbles, en proporciones similares a las observadas en los ensayos (datos no mostrados), puede deducirse que la lacasa solo está involucrada en la remoción de lignina y la solución del búffer puede ser la causante de la lixiviación de los extraíbles $[34,35]$.

Los anterior puede indicar que la composición de extraíbles de los residuos lignocelulósicos del BCA son en su mayoría compuestos solubles en agua, probablemente azúcares remanentes luego de la extracción de caña, así como vitaminas, compuestos nitrogenados (proteicos y no proteicos), ácidos, sustancias fenólicas y cenizas (macro y 

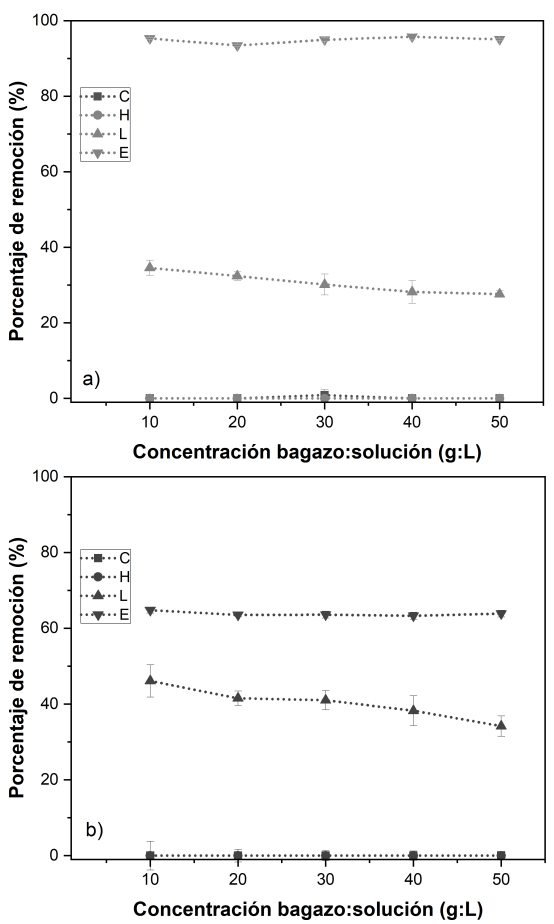

Figura 1. Porcentaje de remoción de los componentes de BCA (a) y BOF (b) a diferentes proporciones bagazo:solución. $\mathrm{C}$ : celulosa, $\mathrm{H}$ : hemicelulosa, L: lignina, E: extraíbles.

microelementos) en menor proporción [36]. Por su parte, la menor remoción observada en el BOF puede atribuirse a la presencia de mucilagos que es un carbohidrato viscoso y complejo, el cual forma redes moleculares que son capaces de captar y retener grandes cantidades de agua, aumentando así la viscosidad de la solución, por lo general, para su extracción se requiere precipitarlas en una solución orgánica o en agua destilada. Adicionalmente, puede presentar mayor cantidad de extraíbles solubles en solventes orgánicos como grasas, resinas y ácidos grasos, ésteres de estos ácidos, cera, fitoesterinas [31].

Cabe destacar que estas sustancias no se pudieron eliminar en los lavados preliminares con agua destilada y la desafección con hipoclorito, debido a que las fibras de bagazo fueron lavadas antes de la molienda fina. Por lo tanto, al reducir de tamaño de partícula a un tamaño menor a $20 \mu \mathrm{m}$, el aumento del área superficial facilitó la solubilidad de los extraíbles.

Frente a la tendencia decreciente, observada entre la proporción bagazo:solución y el porcentaje de remoción de lignina, puede deberse a la viscosidad que afecta la reacción de la enzima con la materia prima de lignocelulosa, principalmente en $\mathrm{BOF}$, debido a la presencia de mucilago alrededor del $4 \%$ y $10 \%$ [37], [38] . En las muestras utilizadas en esta investigación el contenido de mucilago determinado oscila entre el 7\% y 15\% para una muestra de 100 g. Cuanto menor es la concentración de sólidos del sistema de reacción, menor es la viscosidad, lo que conduce a un mayor rendimiento de transferencia de masa y beneficios para la reacción enzimática. Sin embargo, este tipo de tratamientos con enzima lacasa, representa un potencial de aplicación para la exposición del biopolímero de la celulosa cuando se utiliza como pretratamiento previo a un tratamiento con soluciones químicas ya sean alcalinas o ácidas, ya que permite que estas soluciones interactúen con mayor eficiencia con las muestras lignocelulósicas. Además, el uso de este tipo de enzimas inhibe la producción de subproductos tóxicos a diferencia de los tratamientos químicos que generan subproductos que son potenciales contaminantes para el medio ambiente [39, 40].

En este tipo de tratamientos, generalmente, los grupos hidroxilo fenólicos presentes en la lignina se oxidaron para formar quinonas o lignina carbonil por lacasa, lo que da como resultado la degradación de las macromoléculas de lignina [41]. La remoción de la lignina con enzima lacasa, está muy influenciada por las condiciones de temperatura, $\mathrm{pH}$, dosis de la enzima y concentración de sustrato y tipo de enzima a utilizar. Autores como J. Huang et al., informaron una disminución en el contenido de lignina del $21.6 \%$ al $16.2 \%$ con una dosis de lacasa de $120 \mathrm{U} / \mathrm{g}$, temperatura de $50{ }^{\circ} \mathrm{C}$ y un tiempo de pretratamiento de $5 \mathrm{~h}$ en muestras de bagazo de caña [42]. Chen et al., observaron una disminución en el contenido de lignina cuando la dosis de lacasa aumentó de $80 \mathrm{U} / \mathrm{g}$ a $120 \mathrm{U} / \mathrm{g}$, con una temperatura de $70^{\circ} \mathrm{C}$ y un tiempo total de reacción de $5 \mathrm{~h}$ para tratar los tallos de tabaco [43]. Asimismo, Wang et al., disolvieron pulpa de una mezcla de madera que se pretrató con $90 \mathrm{U} / \mathrm{g}$ de lacasa para extraer lignina, con una temperatura de $36{ }^{\circ} \mathrm{C}$. La eficiencia de degradación de la lacasa disminuyó a medida que aumentaba el tiempo de 3 $\mathrm{h}$ a $5 \mathrm{~h}[44]$. 
La Figura 2 muestra la velocidad inicial de remoción de lignina para cada una de las proporciones bagazo: solución evaluadas. Tanto para los ensayos con BCA como para BOF se observó una tendencia ascendente, dentro de las condiciones evaluadas, no alcanzándose un máximo. Esto indica que no hay evidencias de inhibición, pudiéndose aplicar un análisis preliminar bajo la ecuación de MichaelisMenten. Sin embargo, los ensayos con BOF presentaron mayores velocidades que los ensayos con BCA. Probablemente, esto se pueda deber a que la estructura lignocelulósica del BCA presenta una fuerte estructura recalcitrante resistente a la biodegradación de la estructura de la pared celular por ataque enzimático y microbiano y se requiere un proceso de pretratamiento adecuado para facilitar el acceso de las enzimas a los polisacáridos vegetales [45, 46].



Figura 2. Velocidad inicial de remoción de lignina vs. proporción bagazo:solución de BCA y BOF.

Las Tablas 2 y 3 informan los valores de $k_{m}$ y $V_{\max }$ encontrados en cada uno de los métodos de linealización evaluados, a partir de los datos experimentales de los ensayos con BCA y BOF respectivamente. Con Lineweaver-Burk, los resultados de ajuste expresan un coeficiente de correlación (R) mayor a 0.99, tanto para BCA como para BOF, lo que sugiere que las constantes cinéticas obtenidas mostraron una buena predicción en el tratamiento con lacasa. Caso contrario, los ajustes llevados a cabo por Eadie-Hofstee y Agustinson no presentaron un ajuste adecuado, debido a los valores de $\mathrm{R}$ por debajo de 0.90 . En función de ellos se consideró los datos obtenidos por el método de Lineweaver-Burk como válidos para el modelo predictivo de remoción de lignina de los bagazos utilizados.
Tabla 2. Cálculo de $V_{\max }$ y $k_{m}$ a partir de los datos experimentales de proporción lignina:solución obtenidos de los tratamientos de BCA con lacasa

\begin{tabular}{lccc}
\hline Método & $k_{m}\left(g_{\text {lignina }}: \mathbf{L}\right)$ & $V_{\max }\left(g_{\text {lignina }}: \mathbf{L} / \mathbf{h}\right)$ & $\mathbf{R}$ \\
Lineweaver-Burk & 40.2011 & 14.9566 & 0.9933 \\
Eadie-Hofstee & 25.3236 & 10.4648 & 0.7216 \\
Agustinson & 42.6483 & 15.7056 & 0.8003 \\
\hline
\end{tabular}

Tabla 3. Cálculo de $V_{\max }$ y $k_{m}$ a partir de los datos experimentales de proporción lignina:solución obtenidos de los tratamientos de BOF con lacasa

\begin{tabular}{lccc}
\hline Método & $k_{m}\left(g_{\text {lignina }}: \mathbf{L}\right)$ & $V_{\max }\left(g_{\text {lignina }}: \mathbf{L} / \mathbf{h}\right)$ & $\mathbf{R}$ \\
\hline Lineweaver-Burk & 43.5575 & 26.8300 & 0.9972 \\
Eadie-Hofstee & 37.3963 & 23.8118 & 0.7523 \\
Agustinson & 62,6651 & 36.8462 & 0.8005 \\
\hline
\end{tabular}

La linealización de Lineweaver-Burk informó el mejor ajuste de los datos. Sin embargo, presenta algunas limitaciones, al utilizar $[\mathrm{S}]$ relativamente bajas. Así, los valores de las inversas $1 /[\mathrm{S}]$ y $1 / V_{o}$ son grandes y, por lo tanto, también lo son los desvíos ocasionados por los valores experimentales. Tiene la desventaja de comprimir los puntos de datos en concentraciones altas de sustrato en una región pequeña y enfatizar los puntos en concentraciones de sustrato más bajos, que a menudo son los menos precisos. La intersección en el gráfico de Lineweaver-Burk es equivalente a la inversa de $V_{\max }$, por lo que cualquier pequeño error en la medición se magnifica $[47,48]$.

De manera similar, en la linealización de EadieHofstee $V_{0}$ aparece en ambos ejes; por tanto, cualquier error experimental también estará presente en ambos ejes. Además, los errores experimentales o las incertidumbres se propagan de manera desigual y se hacen más grandes sobre la abscisa, lo que da más peso a los valores más pequeños de $V_{0} / S$. Sin embargo, esta linealización es más robusta frente a datos propensos a errores, particularmente porque otorga el mismo peso a los puntos de datos en cualquier rango de concentración de sustrato o velocidad de reacción y los datos que más se desvían de la línea de ajuste son fácilmente observables. Aunque el gráfico produciría en teoría estimaciones más fiables, la presencia de la variable dependiente, la velocidad, en ambos ejes dificulta el análisis riguroso de errores. Con respecto a la linealización 
de Agustinson tiene una mejor distribución de datos entre las tres. Sin embargo, su principal inconveniente es que, de nuevo, ni las ordenadas ni las abscisas representan valores independientes, pues se denota dependencia de la concentración del sustrato y, como resultado, la medida del ajuste, el coeficiente de correlación R, no es aplicable $[49,50]$.

En los parámetros cinéticos informados, los ensayos con BOF presentaron una velocidad máxima $79.38 \%$ mayor que BCA, aunque el valor de $k_{m}$ solo se diferenció en un $7.5 \%$. Lo anterior indica que, aunque la enzima presenta una afinidad similar para los dos sustratos, la eficiencia catalítica $\left(V_{\max } / k_{m}\right)$ es mayor para BOF. Aunque como residuos lignocelulósicos presenten la misma estructura recalcitrante, en donde la celulosa se encuentra cubierta por la lignina y la hemicelulosa, el BCA presenta una fuerte estructura que inhibe la biodegradación de la estructura de la pared celular por ataque enzimático y microbiano, se requiere un proceso de pretratamiento químico adecuado para facilitar el acceso de las enzimas a los polisacáridos de la biomasa.

Algunos compuestos presentes en BOF informan enlaces $\beta$-polisacáridos, es decir, unidades de glucosa unidas $(1 \rightarrow 4)$ - $\beta$ intercaladas con enlaces $(1 \rightarrow 3)-\beta$, que se caracterizan por una estructura de enlace irregular que previene la formación de una estructura cristalina y conduce a una capacidad soluble en agua [51, 52]. Aunque en el BOF la enzima actuó con más facilidad sobre los enlaces fenólicos para eliminar componentes lignocelulósicos, esta remoción pudo ser mayor, pero la viscosidad aumentó inhibiendo así este proceso[53]. Esto no ocurre con los residuos de BCA ya que su estructura se conforma de una amplia gama de fenoles y polifenoles como orto y para-difenoles, fenoles sustituidos con metoxi, ácidos fenólicos y varios otros compuestos donde la enzima no actúa. Estas características estructurales de los fenoles imponen dificultades en su completa degradación y eliminación. La lacasa convierte ciertos compuestos (siringaldehído, ácidos cinámicos, etc.), cataliza la oxidación de los fenoles, genera radicales fenoxi inestables y estos radicales interactúan entre sí, lo que conduce a la polimerización de compuestos aromáticos (que tienen menor capacidad inhibidora) compuestos fenólicos en uno más simple (vainillina), permitiendo así una mayor eliminación de lignina $[54,55]$.

\section{Conclusiones}

En las comparaciones cinéticas, uno de los aspectos más importantes es la estimación de los parámetros reales de la ecuación MM a partir de los datos experimentales. Los parámetros $k_{m}$ y $V_{\max }$ se estimaron utilizando modelos de linealización: Lineweaver-Burk, de Eadie-Hofstee y de Agustinson, los parámetros obtenidos por estos métodos son muy útiles para analizar el comportamiento de las enzimas. Los valores de $k_{m}$ y $V_{\max }$ se midieron dependiendo de las concentraciones de sustrato. El ajuste de los valores a partir de la linealización de Lineweaver-Burk permite describir con alta confiabilidad la cinética enzimática para el rango de valores evaluados, evidenciando buena concordancia estadística de los resultados al utilizar la enzima lacasa. Las linealizaciones de Eadie-Hofstee y de Agustinson a pesar de ser linealizaciones más confiables, están muy influenciadas por $V_{o}$ y la concentración del sustrato, y como resultados los coeficientes encontrados son menores al 0.90 .

A partir del análisis cinético se pudo predecir que la enzima utilizada presentó una mejor afinidad a la remoción de lignina de cactus, lo cual indica que este tipo de proceso tiene una buena perspectiva a futuro para poderse emplear en el tratamiento de este tipo de residuos agroindustriales, para la purificación de celulosa, cuya composición no parece ser afectada bajo las condiciones evaluadas, lo cual es positivo, ya que no se verían pérdidas significativas de este componente de interés. Sin embargo, frente al tiempo de reacción evaluado y la presencia de carga considerable de compuestos no celulósicos no deseados (hemicelulosa, lignina) hace preciso pensar a futuro en buscar alternativas mixtas, por ejemplo, en combinaciones de tratamientos químicos- biológicos, posterior a un tratamiento físico ya que el tamaño de partícula tiene un efecto positivo en la eliminación de lignina, con el fin de que estos procesos combinados sean ambientalmente sostenibles. 


\section{Agradecimientos}

Al laboratorio del Grupo Procesos Ambientalmente Amigables de la Universidad Pedagógica y Tecnológica de Colombia, donde se llevó a cabo la investigación y al Ministerio de Ciencia Tecnología e Innovación (Minciencias), Boyacá Bio, Gobernación de Boyacá, la Universidad Pedagógica y Tecnológica de Colombia (UPTC) y a la Universidad de Antioquia (UdeA) quienes financiaron el proyecto macro "Valorización de residuos agroindustriales de Boyacá, mediante la producción de polímeros superabsorbentes útiles para mejoramiento de suelos áridos", contrato No. 80740-561-2019, de donde se deriva esta investigación.

\section{Referencias}

[1] L. H. C. Mattoso, K. B. R. Teodoro, J. M. Marconcini, T. J. Bondancia, A. C. Corrêa, and E. de M. Teixeira, "Sugarcane bagasse whiskers: Extraction and characterizations", Ind. Crops Prod., Vol. 33, no. 1, p. 63-66, 2010.

[2] K. Cury R, R. Olivero V, L. Chams Ch, Y. Aguas M, and A. Martinez M, "Residuos agroindustriales su impacto, manejo y aprovechamiento", Rev. Colomb. Cienc. Anim. - RECIA, Vol. 9, no. S, p. 122, 2017.

[3] D. M. Escalante H, Orduz J, Zapata J, Cardona M, "Atlas del Potencial Energético de la Biomasa Residual en Colombia”, Bucaramanga, pp, 150, 2011.

[4] C. Sáenz, E. Sepúlveda, and B. Matsuhiro, “Opuntia sp. mucilage's: A functional component with industrial perspectives", J. Arid Environ., Vol. 57, no. 3, p. 275-290, 2004.

[5] R. E. Olivero Verbe, Y. del R. Aguas Mendoza, I. D. Mercado Martínez, D. P. Casas Camargo, and L. E. Montes Gazabón, "Utilización de Tuna (opuntia ficus-indica) como coagulante natural en la clarificación de aguas crudas", $A v$. Investig. en Ing., Vol. 11, no. 1, p. 70, 2014.

[6] B. Mendoza, E. Gómez, E. Hernández, A. Rodríguez, and N. Chavarría, "Elaboración y caracterización de películas biodegradables a partir de mucilago de nopal-caseinato de sodio y mucilago de nopal-pectina", Ciencias Agroquímicas, Handb., p. 129-136, 2014.

[7] H. Vieyra, U. Figueroa-López, A. GuevaraMorales, B. Vergara-Porras, E. San MartínMartínez, and M. Á. Aguilar-Mendez, “Optimized Monitoring of Production of Cellulose Nanowhiskers from Opuntia ficus-indica (Nopal Cactus)", Int. J. Polym. Sci., Vol. 2015, p. 1-8, 2015.

[8] H. C. Arca, L. I. Mosquera-Giraldo, V. Bi, D. $\mathrm{Xu}, \mathrm{L}$. S. Taylor, and K. J. Edgar, "Pharmaceutical Applications of Cellulose Ethers and Cellulose Ether Esters", Biomacromolecules, Vol. 19, no. 7, p. 2351-2376, 2018.

[9] J. Shokri and K. Adibki, "Application of Cellulose and Cellulose Derivatives in Pharmaceutical Industries", Cellul. - Medical, Pharm. Electron. Appl., p, 47-66, 2013.

[10] M. Patchan et al., , "Synthesis and properties of regenerated cellulose-based hydrogels with high strength and transparency for potential use as an ocular bandage", Mater. Sci. Eng. C, Vol. 33, no. 5, p. 3069-3076, 2013.

[11] S. H. Ye, J. Watanabe, Y. Iwasaki, and K. Ishihara, "Antifouling blood purification membrane composed of cellulose acetate and phospholipid polymer", Biomaterials, Vol. 24, no. 23, p. 4143-4152, 2003.

[12] A. L. Buyanov, A. K. Khripunov, A. A. Tkachenko, E. E. Ushakova, and I. V. Gofman, "High-strength biocompatible hydrogels based on poly(acrylamide) and cellulose: Synthesis, mechanical properties and perspectives for use as artificial cartilage", Polym. Sci. Ser. A, Vol. 55, no. 5, p. 302-312, 2013.

[13] M. Iguchi;, S. Yamanaka;, and A. Budhiono; "Bacterial cellulose - a masterpiece of nature's arts", J. Mater. Sci., Vol. 35, no. 2, p. 261-270, 2000 .

[14] Ş. Ciumpiliac et al., , "Modelling of sorbic acid diffusion through bacterial cellulose-based 
antimicrobial films", Chem. Pap., Vol. 66, no. 2, p. 144-151, 2011.

[15] M. A. Hubbe, D. J. Gardner, and W. Shen, "Wettability of cellulosics", BioResources, Vol. 10, no. 4, p. 8657-8749, 2015.

[16] B. B. Hallac and A. J. Ragauskas, "Analyzing cellulose degree of polymerization and its relevancy to cellulosic ethanol", Biofuels, Bioprod. Bioref. vol, 5: pp, 215-225, 2011

[17] S. G. Karp, A. L. Woiciechowski, V. T. Soccol, and C. R. Soccol, "Pretreatment strategies for delignification of sugarcane bagasse: A Review", Brazilian Arch. Biol. Technol., Vol. 56, no. 4, p. 679-689, 2013.

[18] R. Acosta, J. Sanabria, and D. Nabarlatz, "Biomass from colombian agroindustrial activities: Characterization and potential for oligosaccharides production", Chem. Eng. Trans., Vol. 65, p. 667-672, 2018.

[19] D. Watkins, M. Nuruddin, M. Hosur, A. Tcherbi-Narteh, and S. Jeelani, "Extraction and characterization of lignin from different biomass resources", J. Mater. Res. Technol., Vol. 4, no. 1, p. 26-32, 2015.

[20] H. Rabemanolontsoa and S. Saka, "Various pretreatments of lignocellulosics", Bioresour. Technol., Vol. 199, p. 83-91, 2016.

[21] P. Penjumras, R. B. A. Rahman, R. A. Talib, and K. Abdan, "Extraction and Characterization of Cellulose from Durian Rind", Agric. Agric. Sci. Procedia, Vol. 2, p. 237-243, 2014.

[22] Dominic WS Wong, "Structure and action mechanism of ligninolytic enzymes", Appl BiochemBiotechnol, Vol. 157 (2), pp, 174-209, 2009.

[23] U. Moilanen, M. Kellock, S. Galkin, and L. Viikari, "The laccase-catalyzed modification of lignin for enzymatic hydrolysis", Enzyme Microb. Technol., Vol. 49, no. 6-7, p. 492-498, 2011.
[24] L. P. Christopher, B. Yao, and Y. Ji, "Lignin biodegradation with laccase-mediator systems", Front. Energy Res., Vol. 2, no. MAR, p. 1-13, 2014.

[25] M. L. Carvalho et al., , "Kinetic study of the enzymatic hydrolysis of sugarcane bagasse", Brazilian J. Chem. Eng., Vol. 30, no. 3, p. 437447, 2013.

[26] Piñeros-Castro, Aprovechamiento de biomasa lignocelulósica, algunas experiencias., Researchgate, vol no 2014. pp 335. 2016.

[27] E. S. Abdel-Halim, "Chemical modification of cellulose extracted from sugarcane bagasse: Preparation of hydroxyethyl cellulose", Arab. J. Chem., Vol. 7, no. 3, p. 362-371, 2014.

[28] G. Vanitjinda, T. Nimchua, and P. Sukyai, "Effect of xylanase-assisted pretreatment on the properties of cellulose and regenerated cellulose films from sugarcane bagasse", Int. J. Biol. Macromol., Vol. 122, p. 503-516, 2019.

[29] M. Buchanan, "Solvent extractives of wood and pulp (Proposed revision of T $204 \mathrm{~cm}-97$ )", Nanofibers - Prod. Prop. Funct. Appl., p. 6, 2012.

[30] L. H. C. Mattoso, K. B. R. Teodoro, J. M. Marconcini, T. J. Bondancia, A. C. Corrêa, and E. de M. Teixeira, "Sugarcane bagasse whiskers: Extraction and characterizations", Ind. Crops Prod., Vol. 33, no. 1, p. 63-66, 2010.

[31] B. R. A. Alencar, E. D. Dutra, E. V. de S. B. Sampaio, R. S. C. Menezes, and M. A. Morais, "Enzymatic hydrolysis of cactus pear varieties with high solids loading for bioethanol production", Bioresour. Technol., Vol. 250, p. 273-280, 2018.

[32] M. E. Malainine, A. Dufresne, D. Dupeyre, M. Mahrouz, R. Vuong, and M. R. Vignon, "Structure and morphology of cladodes and spines of Opuntia ficus-indica. Cellulose extraction and characterisation", Carbohydr. Polym., Vol. 51, no. 1, p. 77-83, 2003. 
[33] Isael Fuentes Herrera "Extracción de celulosa a partir de Opuntia ficus para la evaluación sobre la retención de flúor (F-)", Mexico, Universidad Autonoma Del Estado de Mexico, Mex p 72, 2018.

[34] L. López, A. Sarmiento, J. Fajardo, L. Valarezo, and R. Zuluaga Gallego, "Determinación del porcentaje de humedad, solubles e insolubles en agua de la fibra de Carludovica Palmata (paja toquilla)", Ingenius, Vol. 9, no. 9, 2013.

[35] A. K. Chandel, S. S. da Silva, W. Carvalho, and O. V. Singh, "Sugarcane bagasse and leaves: Foreseeable biomass of biofuel and bioproducts", J. Chem. Technol. Biotechnol., Vol. 87, no. 1, p. 11-20, 2012.

[36] F. Masarin et al., , "Chemical composition and enzymatic digestibility of sugarcane clones selected for varied lignin content", Biotechnol. Biofuels, Vol. 4, no. 1, p. 55, 2011.

[37] Myrna Alicia Abraján Villaseñor, Efecto en el metodo de extraccion de las caracteristicas quimicas y fisicas del mucilago den nopal ( $O p$ untia ficus-indica) y estudio de su aplicación como recubrimiento comestible,España, Universidad politecnica de valencia,Valencia, Es p. 233, 2008.

[38] Evelyn Ester Jiménez Fernández, “"Obtención del mucílago de la cáscara de la tuna (Opuntia Ficus- Indica) a partir de diferentes métodos de extracción". Chile, Universidad de Chile, Santiago, Chl p. 63, 2014.

[39] L. Mesa, E. González, C. Cara, M. González, E. Castro, and S. I. Mussatto, "The effect of organosolv pretreatment variables on enzymatic hydrolysis of sugarcane bagasse", Chem. Eng. J., Vol. 168, no. 3, p. 1157-1162, 2011.

[40] V. K. Ponnusamy et al., , "A review on lignin structure, pretreatments, fermentation reactions and biorefinery potential", Bioresour. Technol., Vol. 271, p. 462-472, 2019.

[41] X. P. Ouyang, Y. Yang, G. D. Zhu, and X. Q. Qiu, "Radical synthesis of tetrameric lignin model compound", Chinese Chem. Lett., Vol. 26, no. 8, p. 980-982, 2015.

[42] J. Huang, Y. Liu, B. Sun, J. Li, R. Zhang, and S. Nie, "Laccase pretreatment for enhancing microwave-assisted alkaline extraction of hemicellulose from bagasse", BioResources, Vol. 14, no. 1, p. 931-942, 2019.

[43] C. Chen, L. Jiang, G. Ma, D. Jin, L. Zhao, and X. Ouyang, "Lignin Removal from Tobacco Stem with Laccase Improved by Synergistic Action of Weak Alkali and Tween 80", Waste and Biomass Valorization, Vol. 10, no. 11, p. 3343-3350, 2019.

[44] Q. Wang, S. Liu, G. Yang, and J. Chen, "Modeling laccase-induced lignin removal in prehydrolysis liquor from kraft-based dissolving pulp production", Bioresour. Technol., Vol. 175, p. 638-641, 2015.

[45] J. Zhang, H. Zhou, D. Liu, and X. Zhao, Pretreatment of lignocellulosic biomass for efficient enzymatic saccharification of cellulose. $p$. 17-65, 2019

[46] R. M. R. G. Almeida and M. R. Ladisch," Enzyme interactions on lignocellulosic biomass structure". p. 33-59, 2020.

[47] N. V. Bhagavan and C.-E. Ha, "Enzymes and Enzyme Regulation", Essentials Med. Biochem., p. 47-58, 2011.

[48] K. A. Johnson and R. S. Goody, "The original Michaelis constant: Translation of the 1913 Michaelis-Menten Paper", Biochemistry, Vol. 50, no. 39, p. 8264-8269, 2011.

[49] Y. S. Cho and H. S. Lim, "Comparison of various estimation methods for the parameters of Michaelis-Menten equation based on in vitro elimination kinetic simulation data", Transl. Clin. Pharmacol., Vol. 26, no. 1, p. 39-47, 2018.

[50] M. Marasović, T. Marasović, and M. Miloš, "Robust Nonlinear Regression in Enzyme Kinetic Parameters Estimation", J. Chem., Vol. 2017, p. 12, 2017. 
[51] A. Ramezani Kakroodi, S. Panthapulakkal, M. Sain, and A. Asiri, "Cellulose nanofibers from the skin of beavertail cactus, Opuntia basilaris, as reinforcements for polyvinyl alcohol", $J$. Appl. Polym. Sci., Vol. 132, no. 36, p. 1-7, 2015.

[52] S. Bensadón, D. Hervert-Hernández, S. G. Sáyago-Ayerdi, and I. Goñi, "By-Products of Opuntia ficus-indica as a Source of Antioxidant Dietary Fiber", Plant Foods Hum. Nutr., Vol. 65, no. 3, p. 210-216, 2010.

[53] M. Cheikh Rouhou, S. Abdelmoumen, S Thomas, H. Attia, and D. Ghorbel, "Use of green chemistry methods in the extraction of dietary fibers from cactus rackets (Opuntia ficus indica): Structural and microstructural studies", Int. J. Biol. Macromol., Vol. 116, p. 901-910, 2018.
[54] Raquel Gabriela Córdoba Bolaños Germán Ricardo Cultid Chamorro., Estudio comparativo de la actividad enzimática de Lacasa (Lac), lignina peroxidasa (LiP) y Manganeso peroxidasa $(\mathrm{MnP})$ de "pleurotus ostreatus" cultivado en residuos lignocelulosicos de raquis de palma de aceite, bagazo de fique y pulpa de café, Nariño, Universidad de Nariño (Udenar), Pasto, Col, 2015.

[55] Z. Zhu, N. Sathitsuksanoh, T. Vinzant, D. J. Schell, J. D. McMillan, and Y. H. P. Zhang, "Comparative study of corn stover pretreated by dilute acid and cellulose solventbased lignocellulose fractionation: Enzymatic hydrolysis, supramolecular structure, and substrate accessibility", Biotechnol. Bioeng., Vol. 103, no. 4, p. 715-724, 2009. 